\title{
Subtle elbow instability associated with lateral epicondylitis
}

\author{
Sang Ho Kwak', Seung-Jun Lee ${ }^{1 *}$, Hee Seok Jeong ${ }^{2}$, Min Uk Do ${ }^{1}$ and Kuen Tak Suh ${ }^{1}$
}

\begin{abstract}
Background: In lateral epicondylitis, even in the absence of apparent instability, subtle instability can be found under anesthesia. We wanted to ascertain the following: (1) how many elbows surgically treated with lateral epicondylitis showed subtle instability during examination under anesthesia (EUA), (2) how effective magnetic resonance imaging (MRI) was in predicting subtle instability, and (3) if any difference existed in preoperative clinical data between elbows with and without subtle instability during EUA.

Methods: One hundred and twenty-two elbows (117 patients) diagnosed with intractable lateral epicondylitis underwent surgical treatment. No elbow showed apparent instability with conventional physical examination. Under general anesthesia, the elbows were examined for subtle instability via fluoroscopy and divided into unstable and stable groups. Potential prognostic factors and functional scores were assessed retrospectively. The MRls were reviewed again by two radiologists.

Results: Seventeen elbows (unstable group, 13.9\%) had subtle instability in EUA, while 105 elbows (stable group, 86.1\%) did not. Lateral collateral ligament (LCL) complex injury was noted in the MRls of 28 elbows. Fifteen elbows showed subtle instability among 28 elbows with abnormal MRI (positive predictive value, 53.6\%), while 81 elbows did not show subtle instability among 82 elbows with normal MRI (negative predictive value, 98.7\%). The preoperative visual analog scale score was higher in the unstable group than in the stable group $(p<0.001)$, and a history of multiple corticosteroid injections $(\geq 3)$ was related to subtle instability in EUA $(p=0.042)$. Other factors showed no significant differences between both groups.

Conclusions: Subtle instability resulting from LCL complex injury was noted in elbows with lateral epicondylitis. This could be visualized with fluoroscopic EUA, and preoperative MRI could be used to exclude subtle instability. Surgeons should consider checking for subtle instability, especially when patients have a history of multiple corticosteroid injections $(\geq 3)$ or severe pain and MRI indicates instability.
\end{abstract}

Keywords: Lateral, Epicondylitis, Tendinosis, Ligament, Instability, Elbow

\section{Background}

Lateral epicondylitis is usually diagnosed based on clinical history and physical examination. Excluding conditions that can mimic lateral epicondylitis is very important because symptoms cannot be fully relieved if such lesions are neglected. For a differential diagnosis, multiple modalities including simple radiography, ultrasonography, magnetic resonance imaging (MRI), and electrodiagnosis can be used.

\footnotetext{
* Correspondence: Ninanojune@naver.com

${ }^{1}$ Department of Orthopaedic Surgery, Pusan National University Yangsan

Hospital, Pusan National University School of Medicine, 20, Geumo-ro,

Mulgeum-eup, Yangsan-si, Gyeongsangnam-do, Republic of Korea

Full list of author information is available at the end of the article
}

Associated ligament injuries in lateral epicondylitis were reported using MRI or via conventional physical examination. MRI can be used for differentiating other pathologic conditions such as plica, elbow arthritis, and osteochondral defect. In addition, concomitant ligament injuries involving the medial collateral ligament $(\mathrm{MCL})$, lateral collateral ligament (LCL), and lateral ulnar collateral ligament (LUCL) have also been observed in MRI-diagnosed lateral epicondylitis [1, 2]. Associated ligament injuries can induce elbow instability eventually, and posterolateral rotatory instabilities after trauma, corticosteroid injection, and iatrogenic injury during debridement for lateral epicondylitis have been reported [3, 4]. In these reports, most

C The Author(s). 2018 Open Access This article is distributed under the terms of the Creative Commons Attribution 4.0 International License (http://creativecommons.org/licenses/by/4.0/), which permits unrestricted use, distribution, and reproduction in any medium, provided you give appropriate credit to the original author(s) and the source, provide a link to the Creative Commons license, and indicate if changes were made. The Creative Commons Public Domain Dedication waiver (http://creativecommons.org/publicdomain/zero/1.0/) applies to the data made available in this article, unless otherwise stated. 
of the patients showed apparent symptoms including instability via conventional physical examination, feeling of "pop," or prominent swelling of the elbow joint [3, 4]. However, Kalainov revealed that one patient showed unspecific physical examination and the posterolateral instability was detected only during general anesthesia [5]. Morrey et al. described lax LCL and LUCL found in fluoroscopy under local anesthesia or arthroscopic examination as subtle instability, which the authors reported as causes of refractory lateral epicondylitis [4]. Thus, even though conventional physical examination may not reveal associated ligament injuries, subtle instability can be found under specific examination with anesthesia. However, reports of subtle instability found in primary surgery for lateral epicondylitis are very rare, and except for one case [5], only one study reported about subtle instability under anesthesia and suggested a treatment algorithm [6].

In this study, we examined elbow instability in fluoroscopy during examination under anesthesia (EUA) and aimed to report the elbows that needed primary surgical interventions for lateral epicondylitis: (1) how many elbows had subtle instability, (2) how closely the EUA findings matched the MRI and operative findings in elbows with subtle instability, and (3) whether any differences existed regarding preoperative clinical data between elbows with and without subtle instability.

\section{Methods}

This was a retrospective case series study. After approval by our institutional board review (IRB number 05-2017-028), 173 consecutive elbows (168 patients) with lateral epicondylitis treated surgically between March 2011 to December 2016 were enrolled in this study. Definitive criteria for lateral epicondylitis included 1) pain at the elbow during the preceding 30 days and 2) pain at the lateral humeral epicondyle region and pain provoked by resisted extension of the wrist with the elbow extended [7]. All patients were treated with a combination of four conservative methods including NSAID administration, counterforce bracing, isometric exercise, and extensor muscle stretching. Injections including corticosteroid, autologous blood, or botulinum toxin were not given in our protocol. The surgical indication was intractable pain (visual analog scale $[\mathrm{VAS}] \geq 4$ ) after conservative treatment for at least 6 months. Simple radiographs were taken at initial diagnosis, and after deciding to treat with surgery, MRIs were performed. Elbows with previous trauma, including elbow dislocation and fracture $(n=17)$; deformities, including cubitus varus and valgus $(n=11)$; synovial plica and osteochondral defect on MRI $(n=5)$; and previous surgery for lateral epicondylitis $(n=7)$ were excluded. The mediolateral stress, posterolateral rotatory drawer, push-up, and tabletop tests were conducted again by two orthopedic specialists (KSH, LSJ) before surgery. If at least one examiner found any instability in these examinations, the patients $(n=8)$ were considered to have instability during conventional physical examination and excluded. None of the patients showed generalized ligament laxity (Beighton score <4) [8]. Patients detected with synovial plicae intraoperatively $(n=3)$ were also excluded. Finally, 122 elbows (117 patients) were included in this study.

\section{Identifying instability during EUA}

The patients were placed in the supine position under general anesthesia. For valgus instability, the arm was positioned with the elbow in $30^{\circ}$ flexion, humerus in full internal rotation, and forearm in pronation. Then, the manual valgus stress test was performed, and an anteroposterior (AP) image of the elbow was acquired using a fluoroscopic image intensifier [9]. A widening of more than $1 \mathrm{~mm}$ at the ulnohumeral joint was considered as subtle valgus instability [9]. For varus instability, the arm was positioned with the elbow in $15^{\circ}$ flexion, humerus in full external rotation, and forearm in supination. Then, manual varus stress test was performed, and an AP fluoroscopic image was acquired. Since a previous study reported that the radiocapitellar joint is $0.47 \mathrm{~mm}$ more redundant compared with the ulnohumeral joint [10], a widening of over $1.5 \mathrm{~mm}$ at the radiocapitellar joint was considered as subtle varus instability (Fig. 1). In addition, a posterolateral pivot shift test was performed with the elbow in $90^{\circ}$ flexion and forearm in supination [11]. If the longitudinal axis of the radius does not pass through the center of the capitellum, it was considered as a posterolateral instability [9]. Thereafter, a lateral image of the elbow was acquired to identify subtle posterolateral instability (Fig. 2a-c) [5]. If subtle instability was found, the contralateral asymptomatic elbow was examined to exclude underlying normal laxity of elbow joint.

\section{Surgical exploration of ligament status}

During surgery, intraarticular pathology was checked via arthrotomy, and 3 patients with synovial plicae were excluded. For patients without subtle instability, we performed open release of lateral epicondylitis. The LCL complex was not explored to minimize damage to the extensor tendon. For patients with subtle instability, additional exploration for unstable ligament was performed. For varus or posterolateral instability, the proximal LCL complex was explored by elevating the common extensor origin, and the distal LCL complex was explored by elevating the anconeus and extensor carpi ulnaris. For valgus instability, the MCL was explored via an additional medial longitudinal incision.

\section{Preoperative clinical data and MRI evaluation}

Demographic data that were considered as potential prognostic factors such as patient age, gender, duration of education, and involvement of the dominant hand were assessed retrospectively. Other potential prognostic factors 


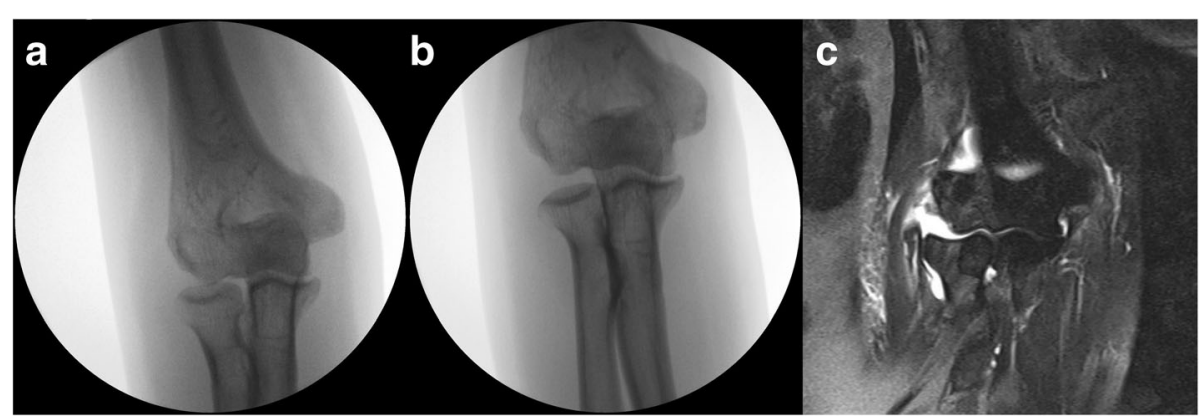

Fig. 1 a Intraoperative fluoroscopy image of a 53-year-old woman (patient \#1). b Widening of the radiocapitellar joint was identified $2.3 \mathrm{~mm}$ in the varus stress view. c T2-weighted coronal magnetic resonance image showed rupture of the lateral collateral ligament and tear of the extensor tendon origin

such as duration of symptoms, smoking, body mass index (BMI), and history of multiple corticosteroid injections $(\geq 3)$ were also assessed [7, 12-15]. Preoperatively, VAS score, Mayo Elbow Performance Score (MEPS), and Quick Disabilities of the Arm, Shoulder, and Hand (Quick DASH) score were assessed by an experienced orthopedic surgeon who did not attend the surgery. To identify ligament lesions in MRI, two radiologic specialists (TYM, HSJ), who had no knowledge of the intraoperative findings, reviewed the MRIs again.

\section{Statistical analysis}

Data were presented as mean \pm standard deviation (SD). Categorical variables were analyzed using Fisher's exact test, and continuous variables were analyzed using the Mann-Whitney test. Statistical significance was defined as a $P$-value $<0.05$. Data were analyzed with SPSS for Windows version 18.0 (SPSS, Chicago, Illinois, USA).

\section{Results}

Of the 122 elbows (117 patients), 17 elbows (16 patients) with subtle instability were assigned to the unstable group during EUA. One hundred and five elbows (101 patients) without instability were assigned to the stable group. All 17 elbows in the unstable group showed varus instability (radiocapitellar joint widening; range, 2.5 4. $1 \mathrm{~mm}$ ), and two showed additional posterolateral instability. No elbow in the unstable group showed valgus

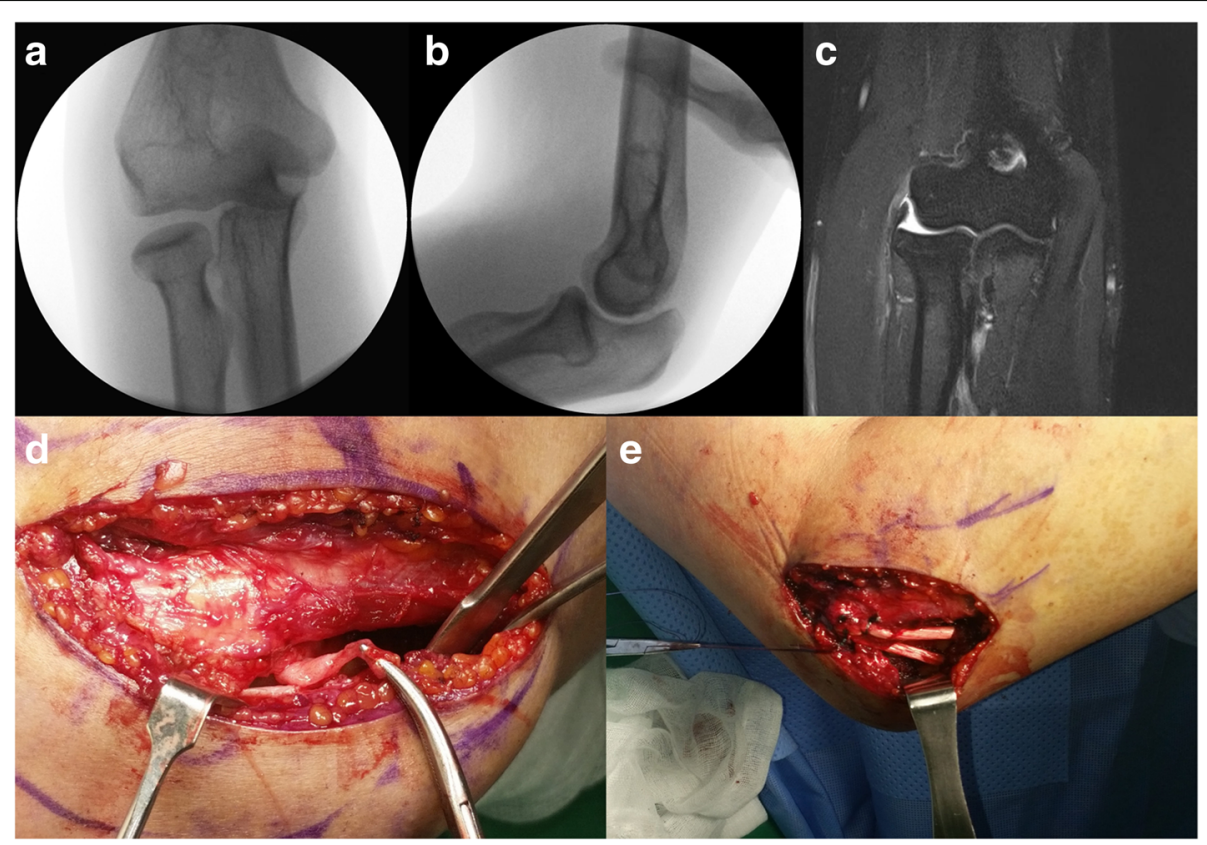

Fig. 2 a The $2.1 \mathrm{~mm}$ widening of the radiocapitellar joint was identified in the varus stress view of a 38-year-old man (patient \#5). $\mathbf{b}$ The radial head was shifted posteriorly in the posterolateral pivot shift test. c T2-weighted coronal magnetic resonance image showed rupture of the lateral collateral ligament. $\mathbf{d}$ Intraoperatively, the definite lateral and lateral ulnar collateral ligaments were not found, except for thin fibrous tissue. e The lateral ulnar collateral ligament was reconstructed with a tibialis anterior allograft 
instability under EUA (ulnohumeral joint widening; range, $0.13 \sim 0.66 \mathrm{~mm}$ ). Except for 1 patient in the unstable group (patient 2), there was no patient who showed subtle instability on the contralateral asymptomatic elbow. In 105 elbows without subtle instability, the radiocapitellar and ulnohumeral joint widening was measured, ranging from 0.45 to $1.32 \mathrm{~mm}$ and 0.15 to 0 . $75 \mathrm{~mm}$, respectively.

One elbow in the unstable group and 11 elbows in the stable group did not undergo MRI for economic reason. Among 16 elbows which underwent MRI in the unstable group, 4 LCL + LUCL injuries, 6 LUCL injuries, and 5 LCL injuries were found. In 1 patient (patient 8), there was no notable ligament tear except for signal change on MRI taken preoperatively. In the stable group, 94 elbows underwent MRI, and $8 \mathrm{LCL}$ and 5 LUCL injuries were noted on MRI. A combined LCL + LUCL injury was not identified. In both groups, no MCL injury was noted with MRI. Of all 28 elbows with LCL complex injuries on MRI, 15 showed subtle instability with EUA. Intraoperatively, in the unstable group, LCL complex abnormalities were confirmed at surgery in all cases; no functioning LCL and LUCL structure except the annular ligament was considered as a deficient LCL complex $(n=8)$ and remained a functioning structure despite that the defect or attenuation was considered as a partial loss of the LCL complex $(n=9)$ (Fig. 3). Capsules were not torn but attenuated in all patients (Table 1).

Duration of symptoms, gender, age, dominant hand involvement, occupation, educational periods, smoking, BMI, preoperative MEPS, and preoperative Quick DASH score were not different between both groups. The multiple corticosteroid injection rate $(\geq 3)$ and preoperative VAS score were higher in the unstable group than in the stable group (Table 2).

\section{Discussion}

An elbow with significant instability can be detected via conventional physical examinations. However, it is difficult to demonstrate chronic ligament injury of subtle instability alone in the awake patient because of muscular restraints. Thus, EUA with muscle relaxation should be performed in these patients $[16,17]$. On EUA, pathologic gapping of the lateral joint in the AP stress view or posterior translation of the radial head in the lateral stress view may provide evidence of lateral elbow instabilities and was applied in the current study [16]. Previously, EUA was not usually performed in lateral epicondylitis, and only arthroscopic EUA was reported [6]. In this study of 40 patients, 13 patients with dehiscence between 3 and $6 \mathrm{~mm}$ showed slight instability, and 2 patients with dehiscence over $6 \mathrm{~mm}$ showed severe instability. Arthroscopic examination had some potential advantages because there was no need for radiation and it was easy to perform anytime during the surgery. However, this procedure had a disadvantage in that surgeons could identify dehiscence on varus or valgus stress tests alone and obtain little information about posterolateral instability. Moreover, joint distension with saline can influence the tension of the capsule and ligament, causing some difficulties in analyzing the stability. Fluoroscopic EUA poses a risk of radiation exposure, and it might be difficult to note minor instability. However, it is possible to distinguish posterolateral instability from simple coronal instability, and it is easy to perform without any special technique. Moreover, it can provide more intuitive images of instability. Considering these advantages and disadvantages, the surgeon must decide which method to choose in identifying instability in EUA.

While few reports have been published about combined instability in EUA, there are several reports about associated ligament injuries with lateral epicondylitis identified via MRI. Potter et al. reported that 4 of 20 patients had associated radial collateral ligament injuries with MRI and those lesions were confirmed during surgery [17]. More recent studies have reported a positive correlation between the severity of common extensor tendon injury and that of LUCL injury $[1,2]$. Moreover, in a study comparing 9 patients and 9 asymptomatic volunteers, MRI was reported to be effective in determining ligament abnormality in patients with subtle elbow

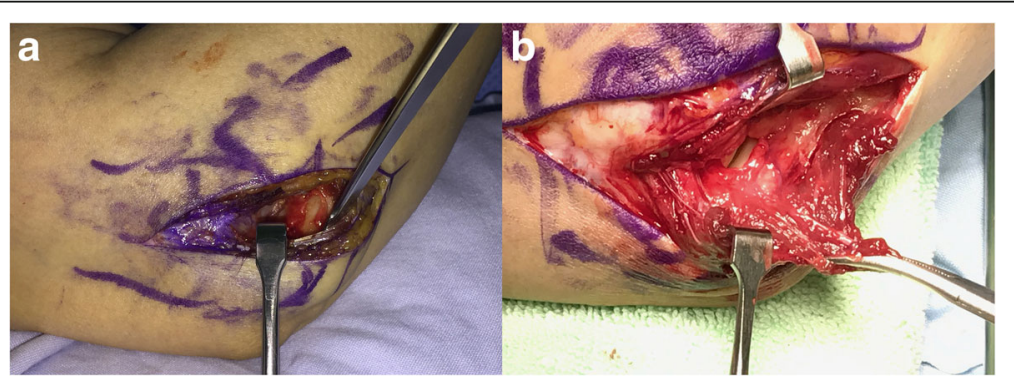

Fig. 3 a Example of a deficient $L C L$ complex: no visible functioning $L C L$ complex was found. $\mathbf{b}$ Example of partial defect of the $L C L$ complex: there was a defect in the $L C L$ complex, but a functional $L C L$ complex partially remained 
Table 1 Patient demographics, findings in MRI and EUA, and complication in unstable group

\begin{tabular}{|c|c|c|c|c|c|c|c|c|}
\hline Patient & Gender & $\begin{array}{l}\text { Age } \\
\text { (decade) }\end{array}$ & Handedness & $\begin{array}{l}\text { Duration of } \\
\text { symptom }\end{array}$ & $\begin{array}{l}\text { Multiple Injection } \\
\text { history }\end{array}$ & $\begin{array}{l}\text { Instability under } \\
\text { EUA }\end{array}$ & MRI & $\begin{array}{l}\text { Intraoperative findings of } \mathrm{LCL} \\
\text { complex }\end{array}$ \\
\hline 1 & $\mathrm{~F}$ & 6th & $\operatorname{Rt}(D)$ & 7 & + & Var & $\begin{array}{l}\mathrm{LCL}, \\
\mathrm{LUCL}\end{array}$ & Deficient \\
\hline \multirow[t]{2}{*}{2} & $\mathrm{~F}$ & 6th & $\operatorname{Rt}(\mathrm{D})$ & 24 & + & Var & LUCL & Deficient \\
\hline & & & $\operatorname{Lt}(N D)$ & 24 & + & Var & LUCL & Partial defect \\
\hline 3 & M & 6th & $\operatorname{Rt}(\mathrm{D})$ & 36 & + & Var, & $\mathrm{LCL}$ & Deficient \\
\hline 4 & M & 5 th & $\operatorname{Rt}(\mathrm{D})$ & 6 & - & Var, Posterolat & $\begin{array}{l}\mathrm{LCL} \\
\mathrm{LUCL}\end{array}$ & Partial defect \\
\hline 5 & M & 4th & $\operatorname{Rt}(\mathrm{D})$ & 24 & + & Var, Posterolat & LUCL & Deficient \\
\hline 6 & $\mathrm{~F}$ & 6th & $\operatorname{Rt}(\mathrm{D})$ & 12 & + & Var & LUCL & Partial defect \\
\hline 7 & F & 7th & $\operatorname{Lt}(\mathrm{ND})$ & 12 & + & Var & LUCL & Deficient \\
\hline 8 & M & 5 th & $\operatorname{Rt}(\mathrm{D})$ & 10 & + & Var & intact & Partial defect \\
\hline 9 & F & 5 th & $\operatorname{Lt}(\mathrm{ND})$ & 14 & + & Var & $\mathrm{LCL}$ & Partial defect \\
\hline 10 & F & 5 th & $\operatorname{Lt}(\mathrm{ND})$ & 12 & + & Var & LUCL & Deficient \\
\hline 11 & F & 6th & $\operatorname{Rt}(\mathrm{D})$ & 60 & + & Var & $\mathrm{LCL}$ & Deficient \\
\hline 12 & M & 5th & $\operatorname{Rt}(\mathrm{D})$ & 10 & + & Var & $\mathrm{LCL}$ & Partial defect \\
\hline 13 & $F$ & 5 th & $\operatorname{Rt}(D)$ & 12 & + & Var & $\begin{array}{l}\text { Not } \\
\text { taken }\end{array}$ & Partial defect \\
\hline 14 & M & 6th & $\operatorname{Lt}(\mathrm{ND})$ & 14 & + & Var & $\mathrm{LCL}$ & Partial defect \\
\hline 15 & M & 5 th & $\operatorname{Rt}(\mathrm{D})$ & 12 & + & Var & $\begin{array}{l}\mathrm{LCL} \\
\mathrm{LUCL}\end{array}$ & Partial defect \\
\hline 16 & M & 4th & $\operatorname{Rt}(\mathrm{D})$ & 12 & + & Var, Posterolat & $\begin{array}{l}\mathrm{LCL}, \\
\mathrm{LUCL}\end{array}$ & Deficient \\
\hline
\end{tabular}

EUA Examination under anesthesia, MRI Magnetic resonance image, LCL Lateral collateral ligament, LUCL Lateral ulnar collateral ligament

instability [18]. Thus, when suspected, it would be helpful to perform an MRI of the elbow. However, in elbows with lateral epicondylitis, MRI was not completely correlated with EUA and operative findings. In our study, 15 of the 28 abnormal elbow MRIs showed subtle instability (positive predictive value, 53.6\%), while 81 of the 82 normal MRIs did not show subtle instability (negative predictive value, $98.7 \%$ ). Considering the relatively low positive and high negative predictive value, we think MRI alone is not recommended in determining the surgical procedure, and EUA should be performed when there are ligament abnormalities in MRI.

It is clear that an unstable elbow with significant instability should be stabilized because detrimental

Table 2 Comparison of preoperative clinical data. Continuous variables were presented as mean (standard deviation)

\begin{tabular}{llll}
\hline & Unstable Group & Stable Group & P-value \\
\hline Gender(M/F) & $8 / 9$ & $45 / 60$ & 0.473 \\
Age (year) & $48.9(6.61)$ & $50.5(7.47)$ & 0.392 \\
Dominant/Nondominant & $12 / 5$ & $65 / 40$ & 0.344 \\
Manual worker(+/-) & $9 / 8$ & $46 / 59$ & 0.329 \\
Duration (month) & $17.7(13.3)$ & $14.5(10.3)$ & 0.321 \\
Eductaion (year) & $12.6(2.18)$ & $11.9(2.37)$ & 0.275 \\
Multiple corticosteroid injection(> 3)(+/-) & $16 / 1$ & $76 / 29$ & $0.042^{*}$ \\
Smoking(+/-) & $7 / 10$ & $29 / 75$ & 0.195. \\
BMl & $23.6(2.16)$ & $24.3(3.21)$ & 0.373 \\
Pre VAS & $7.29(0.99)$ & $5.48(1.20)$ & $<0.001^{*}$ \\
Pre MEPS & $59.1(5.37)$ & $61.8(5.93)$ & 0.061 \\
Pre Quick DASH & $56.4(6.49)$ & $54.6(7.06)$ & 0.333 \\
\hline
\end{tabular}

VAS Visual analogue scale, MEPS Mayo Elbow Performance index Score, Quick DASH Quick Disabilities of the Arm, Shoulder and Hand Score ${ }^{*} P<0.05$ 
articular contact in the elbow joint usually leads to irreversible cartilage injury [19]. However, whether subtle instability during EUA should be surgically treated is controversial. Aoki et al. reported that a patient with mild lateral instability had a good outcome even with conventional debridement [20]. Coleman et al. reported that synovial fistulae developed in 2 patients postoperatively [21]. One patient with an irreparable defect in the elbow capsule during surgery had to undergo an anconeus flap operation, and the other was managed conservatively. There was no mention about instability in these 2 patients. However, Kalainov et al. reported that 3 patients with large fistulae with instability had to undergo LUCL reconstruction [5]. Moreover, several authors have recommended surgical correction for instability, which was only identified in EUA [16, 18]. We believe that such instabilities, identified during EUA, should preferably be corrected; however, evidence supporting this proposition is still lacking. It might also be unclear which procedure should be selected for subtle instability. Ligament repair, including imbrication or tensioning, could be an option [22]; however, ligamentous tissue often showed poor quality in cases of chronic posterolateral instability, and ligament reconstruction might provide more consistent results [18, 23, 24]. In our study, 13 patients underwent ligament reconstruction because ligament repair was not feasible. Thus, if surgeons decide to address subtle instability, they should prepare for ligament reconstruction as well as repair.

We investigated preoperative factors that were known to affect the prognosis of lateral epicondylitis, to determine which of the preoperative clinical data was different between the two groups. In conservative treatments, demographic factors including age and gender were reported to have no significant relationship with the prognosis of lateral epicondylitis, while the effect of site involvement was unclear. Manual work, high strain at work, long duration of symptoms, multiple musculoskeletal complaints, and low socioeconomic status were reported as poor prognostic factors at 12 months of treatment [7, 12, 14]. After surgical treatments, high baseline pain, sudden onset of symptoms, long duration, morbid obesity, smoking, a history of multiple corticosteroid injections $(\geq 3)$, and young age were reported as potential risk factors for poor surgical outcome [13, 15]. The effect of gender on outcome was considered controversial [13, 25]. Some authors even mentioned previous corticosteroid injection as a cause of ligament tear [5, 26 , and a history of multiple corticosteroid injections $(\geq 3)$ was reported as the most significant risk factor for surgical treatment failure [17]. Among those factors, our results suggest that a high level of pain and history of multiple corticosteroid injections $(\geq 3)$ are preoperative characteristics of lateral epicondylitis with subtle instability. However, patient's recollection of how many injections they received was inaccurate in our study, so we could not perform analysis based on the exact number of injections. Moreover, since the VAS score did not indicate the absolute value of pain, it was difficult to provide cut-off values for pain level predictive of subtle instability.

The present study revealed that some patients $(13.9 \%$ of the patients that underwent surgery for chronic lateral epicondylitis) showed varus or posterolateral subtle instability during fluoroscopic EUA. To our knowledge, our study is the first to report the predictive value of MRI for subtle instability. Nevertheless, our study had several limitations. First, the number of patients in the unstable group was relatively small because subtle instability in EUA is uncommon in lateral epicondylitis. Therefore, results such as risk ratio could not be presented. Second, the force applied during the stress test was not identical. Thus, it was impossible to analyze the joint widening quantitatively. Third, exploration of the LCL complex was not performed in the stable group. Thus, the relationship between the structural status of ligaments and MRI findings was not revealed in this study. Further study should include a larger number of patients and the same force for stress test to overcome these limitations.

\section{Conclusion}

Subtle instability resulting from chronic LCL complex injury was noted in elbows with lateral epicondylitis. This is difficult to detect in conventional physical examination but can be easily visualized in fluoroscopic EUA. Preoperative MRIs can be used to exclude subtle instability with its high negative predictive value. The unstable group tended to have higher VAS scores than the stable group, and a history of multiple corticosteroid injections ( $\geq 3)$ was an indication of subtle instability. Although it was unclear whether surgical treatment of the LCL complex should be performed, surgeons should consider checking for subtle instability, especially when patients have a history of multiple corticosteroid injections $(\geq 3)$ or severe pain and MRI indicates instability.

\footnotetext{
Abbreviations

BMI: Body mass index; ECRB: Extensor carpi radialis brevis; EUA: Examination under anesthesia; LCL: Lateral collateral ligament; LUCL: Lateral ulnar collateral ligament; MEPS: Mayo Elbow Performance Score; MRI: Magnetic resonance imaging; Quick DASH: Quick Disabilities of the Arm, Shoulder, and Hand; SD: Standard deviation; VAS: Visual analogue scale
}

\section{Acknowledgements}

We thank Tae Yong Moon for his help with the radiographic review.

\section{Availability of data and materials}

The datasets generated during and analyzed during the current study are available from the corresponding author on reasonable request. 


\section{Authors' contributions}

SK, SL, and KS have contributed to the conception of the study, acquisition of data, or analysis of data. $\mathrm{HJ}$ contributed to the analysis of image data. MD, SK, and SL were involved in drafting the manuscript and revising it critically. All authors have read and approved the final version of the manuscript.

\section{Ethics approval and consent to participate}

This retrospective study was approved by the ethical review committee, Pusan National University Yangsan Hospital (05-2017-028). Written informed consent was obtained from participants for research studies and presented data.

\section{Competing interests}

The authors declare that they have no competing interests.

\section{Publisher's Note}

Springer Nature remains neutral with regard to jurisdictional claims in published maps and institutional affiliations.

\section{Author details}

'Department of Orthopaedic Surgery, Pusan National University Yangsan Hospital, Pusan National University School of Medicine, 20, Geumo-ro, Mulgeum-eup, Yangsan-si, Gyeongsangnam-do, Republic of Korea. ${ }^{2}$ Department of Radiology, Pusan National University Yangsan Hospital, Pusan National University School of Medicine, 20, Geumo-ro, Mulgeum-eup, Yangsan-si, Gyeongsangnam-do, Republic of Korea.

Received: 20 February 2018 Accepted: 30 April 2018

Published online: 07 May 2018

\section{References}

1. Bredella M, Tirman P, Fritz R, Feller J, Wischer T, Genant H. MR imaging findings of lateral ulnar collateral ligament abnormalities in patients with lateral epicondylitis. AJR Am J Roentgenol. 1999:173(5):1379-82.

2. Qi L, Zhu Z-F, Li F, Wang R-F. MR imaging of patients with lateral epicondylitis of the elbow: is the common extensor tendon an isolated lesion? PLoS One. 2013;8(11):e79498.

3. Dzugan SS, Savoie FH, Field LD, O'Brien MJ, You Z. Acute radial ulnohumeral ligament injury in patients with chronic lateral epicondylitis: an observational report. J Shoulder Elb Surg. 2012;21(12):1651-5.

4. Morrey BF. Reoperation for failed surgical treatment of refractory lateral epicondylitis. J Shoulder Elb Surg. 1992;1(1):47-55.

5. Kalainov DM, Cohen MS. Posterolateral rotatory instability of the elbow in association with lateral epicondylitis. J Bone Joint Surg Am. 2005;5:1120-5.

6. Kniesel B, Huth J, Bauer G, Mauch F. Systematic diagnosis and therapy of lateral elbow pain with emphasis on elbow instability. Arch Orthop Trauma Surg. 2014;134(12):1641-7.

7. Haahr J, Andersen J. Physical and psychosocial risk factors for lateral epicondylitis: a population based case-referent study. Occup Environ Med. 2003;60(5):322-9.

8. Clinch J, Deere K, Sayers A, Palmer S, Riddoch C, Tobias JH, Clark EM. Epidemiology of generalized joint laxity (hypermobility) in fourteen-year-old children from the UK: a population-based evaluation. Arthrits Rheum. 2011;63(9):2819-27.

9. O'Driscoll S. Elbow instability. Acta Orthop Belg. 1999;65(4):404-15.

10. Lee RK, Griffith JF, Yuen BT, Ng AW, Yeung DK. Elbow MR arthrography with traction. Br J Radiol. 2016;89(1064):20160378

11. O'driscoll S, Bell D, Morrey B. Posterolateral rotatory instability of the elbow. J Bone Joint Surg Am. 1991;73(3):440-6.

12. Bot SD, van der Waal JM, Terwee C, Van der Windt D, Bouter LM, Dekker J. Course and prognosis of elbow complaints: a cohort study in general practice. Ann Rheum Dis. 2005;64(9):1331-6.

13. Degen RM, Cancienne JM, Camp CL, Altchek DW, Dines JS, Werner BC. Three or more preoperative injections is the most significant risk factor for revision surgery after operative treatment of lateral epicondylitis: an analysis of 3863 patients. J Shoulder Elb Surg. 2017;26(4):704-9.

14. Smidt N, Lewis M, Windt DAVD, Hay EM, Bouter LM, Croft P. Lateral epicondylitis in general practice: course and prognostic indicators of outcome. J Rheumatol. 2006;33(10):2053-9.

15. Solheim E, Hegna J, Øyen J. Extensor tendon release in tennis elbow: results and prognostic factors in 80 elbows. Knee Surg Sports Traumatol Arthrosc. 2011;19(6):1023-7.
16. Cohen MS. Lateral collateral ligament instability of the elbow. Hand Clin. 2008;24(1):69-77.

17. Potter HG, Hannafin JA, Morwessel RM, DiCarlo EF, O'Brien SJ, Altchek DW. Lateral epicondylitis: correlation of MR imaging, surgical, and histopathologic findings. Radiology. 1995;196(1):43-6.

18. Potter HG, Weiland AJ, Schatz JA, Paletta GA, Hotchkiss RN. Posterolateral rotatory instability of the elbow: usefulness of MR imaging in diagnosis. Radiology. 1997;204(1):185-9.

19. Ring D, Jupiter JB. Reconstruction of posttraumatic elbow instability. Clin Orthop Relat Res. 2000;370:44-56.

20. Aoki M, Wada T, Isogai S, Kanaya K, Aiki H, Yamashita T. Magnetic resonance imaging findings of refractory tennis elbows and their relationship to surgical treatment. J Shoulder Elb Surg. 2005;14(2):172-7.

21. Coleman B, Quinlan JF, Matheson JA. Surgical treatment for lateral epicondylitis: a long-term follow-up of results. J Shoulder Elb Surg. 2010;19(3):363-7.

22. Fedorka CJ, Oh LS. Posterolateral rotatory instability of the elbow. Curr Rev Musculoskelet Med. 2016;9(2):240-6.

23. Charalambous C, Stanley J. Posterolateral rotatory instability of the elbow. J Bone Joint Surg Br. 2008;90(3):272-9.

24. Sanchez-Sotelo J, Morrey B, O'driscoll S. Ligamentous repair and reconstruction for posterolateral rotatory instability of the elbow. J Bone Joint Surg Br. 2005:87(1):54-61.

25. Svernlöv B, Adolfsson L. Outcome of release of the lateral extensor muscle origin for epicondylitis. Scand J Plast Reconstr Surg Hand Surg. 2006:40(3):161-5.

26. Jones KJ, Dodson CC, Osbahr DC, Parisien RL, Weiland AJ, Altchek DW, Allen $\mathrm{AA}$. The docking technique for lateral ulnar collateral ligament reconstruction: surgical technique and clinical outcomes. J Shoulder Elb Surg. 2012;21(3):389-95.

\section{Ready to submit your research? Choose BMC and benefit from:}

- fast, convenient online submission

- thorough peer review by experienced researchers in your field

- rapid publication on acceptance

- support for research data, including large and complex data types

- gold Open Access which fosters wider collaboration and increased citations

- maximum visibility for your research: over $100 \mathrm{M}$ website views per year

At BMC, research is always in progress.

Learn more biomedcentral.com/submissions 